\title{
Extension of paths and cycles for hypergraphs
}

\author{
Gyula Y. Katona ${ }^{1}$ \\ Department of Computer Science and Information Theory \\ Budapest University of Technology and Economics \\ 1521 Budapest Pob. 91, Hungary
}

\begin{abstract}
In [5] we defined the hamiltonian cycle in hypergraphs in a new way. The definition can be extended to paths and cycles as well. There are many results for hypergraphs that uses the traditional path and cycle definition of Berge, so almost all of them can be considered with the new definition. In the present talk, I will deal with such results.
\end{abstract}

Keywords: hamiltonian cycle, tight path, tight cycle

\section{Hamiltonian cycles}

Let $\mathcal{H}$ be a $k$-uniform hypergraph on the vertex set $V(\mathcal{H})=\left\{v_{1}, v_{2}, \ldots, v_{n}\right\}$ where $n>k$. $v_{n+x}$ with $x \geq 0$ denotes the same vertex as $v_{x}$ for simplicity of notation. The set of the edges, $k$-element subsets of $V$, is denoted by $\mathcal{E}(\mathcal{H})=\left\{E_{1}, E_{2}, \ldots, E_{m}\right\}$.

In [5] we gave the following definition:

Definition 1.1 A cyclic ordering $\left(v_{1}, v_{2}, \ldots, v_{n}\right)$ of the vertex set is called a hamiltonian cycle iff for every $1 \leq i \leq n$ there exists an edge $E_{j}$ of $\mathcal{H}$ such that $\left\{v_{i}, v_{i+1}, \ldots, v_{i+k-1}\right\}=E_{j}$.

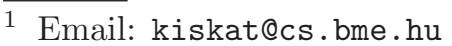


Since an ordinary graph is a 2-uniform hypergraph, this definition gives the definition of the hamiltonian cycle in ordinary graphs for $k=2$. (As a matter of fact, in the original paper the term chain was used instead of cycle, but it seems that everyone prefers cycle.)

The first natural question was to find a Dirac type theorem for hamiltonian cycles. For this, we need to extend the definition of degree for hypergraphs.

Definition 1.2 The degree of a fixed $l$-tuple of distinct vertices, $\left\{v_{1}, \ldots, v_{l}\right\}$, in a $k$-uniform hypergraph is the number of edges of the hypergraph containing all $\left\{v_{1}, v_{2}, \ldots, v_{l}\right\}$. It is denoted by $d^{(k)}\left(v_{1}, v_{2}, \ldots, v_{l}\right)$. Furthermore $\delta_{l}^{(k)}(\mathcal{H})$ denotes minimum of $d^{(k)}\left(v_{1}, v_{2}, \ldots, v_{l}\right)$ over all l-tuples in $\mathcal{H}$. (If $k$ is clear from the context, only $\delta_{l}(\mathcal{H})$ will be used.)

We conjectured that $\delta_{k-1}^{(k)}(\mathcal{H}) \geq\left\lfloor\frac{n-k+3}{2}\right\rfloor$ implies the existence of the hamiltonian cycle, and showed that this bound cannot be lowered. From the other side, a Dirac type theorem was proved for any $k$, however, the degree bound was far from being best possible.

Theorem $1.3([5])$ If $\mathcal{H}=(V, \mathcal{E})$ is a $k$-uniform hypergraph on $n$ vertices with

$$
\delta_{k-1}(\mathcal{H})>\left(1-\frac{1}{2 k}\right) n+4-k-\frac{2}{k},
$$

then $\mathcal{H}$ contains a hamiltonian cycle.

For $k=3$ the above result requires roughly $\frac{5}{6} n$ degree bound for each pair of vertices, but it is conjectured that only $\frac{1}{2} n$ is needed.

Now, more then a decade later, the problem is nearly settled. In [6] Ruciński, Rödl and Szemerédi proved that the conjecture is asymptotically true for $k=3$, then in [8] the exact result was given in this case, together with the analogous hamiltonian path result.

Theorem $1.4([8])$ Let $\mathcal{H}=(V, \mathcal{E})$ be a 3-uniform hypergraph on $n$ vertices where $n$ is sufficiently large. If $\delta_{2}(\mathcal{H}) \geq\left\lfloor\frac{1}{2} n\right\rfloor$ then $\mathcal{H}$ contains a hamiltonian cycle. If $\delta_{2}(\mathcal{H}) \geq\left\lfloor\frac{1}{2} n\right\rfloor-1$ then $\mathcal{H}$ contains a hamiltonian path.

For larger $k$ values only the asymptotically sharp bound is known.

Theorem $1.5([7])$ Let $k \geq 3, \gamma>0$, and let $\mathcal{H}=$ be a $k$-uniform hypergraph on $n$ vertices where $n$ is sufficiently large. If $\delta_{k-1}(\mathcal{H}) \geq\left(\frac{1}{2}+\gamma\right) n$, then $\mathcal{H}$ contains a hamiltonian cycle.

The proofs of the above results uses similar ideas that appear in the proof of the celebrated Szemerédi Lemma, but as a result, the proof works only if $n$ is sufficiently large. Although this lower bound on $n$ is not as large as in 
the original Szemerédi Lemma, but is is still beyond "normal size". So the problem is still open for normal size hypergraphs, as well, as the exact degree bound for larger $k$.

Problem 1.6 Prove that $\delta_{k-1}(\mathcal{H}) \geq\left\lfloor\frac{n-k+3}{2}\right\rfloor$ implies the existence of a hamiltonian cycle for all $k \geq 4$.

\section{Extremal questions}

The first natural extremal question, which already appeared in [5], asks for the maximum number of edges in a uniform hypergraph containing no hamiltonian cycle.

For graphs the extremal case is a complete graph on $(n-1)$ vertices completed by a vertex of degree one. It is trivial to prove that this graph does not contain a hamiltonian cycle, but it is not straightforward to prove that this is the extremal case.

For hypergraphs, even determining the extremal case looks more difficult. An easy double-counting argument gives an upper bound, we count how many hamiltonian cycles are destroyed if an edge is deleted from the complete hypergraph. With Frankl in [2] we improved this upper bound by extending the counting argument. We count how many subhypergraphs are there in the complete hypergraph, that contain a hamiltonian cycle, and at least 2 edges need to be removed to destroy all hamiltonian cycles in this subgraph. Determining the minimum number of edges in such hypergraphs leads to the following improvement of the upper bound.

Theorem 2.1 ([2]) If $\mathcal{H}$ is a $k$-uniform hypergraph on $n$ vertices satisfying

$$
|\mathcal{E}(\mathcal{H})| \geq\left(\begin{array}{l}
n \\
r
\end{array}\right)\left(1-\frac{4 k}{(4 k-1) n}\right)
$$

then $\mathcal{H}$ contains a hamiltonian $(k-1)$-cycle.

In the general case the best construction is due to Tuza [9]. Using Steiner systems he constructed hypergraphs with no hamiltonian cycles and

$$
|\mathcal{E}(\mathcal{H}(n, k))|=\left(\begin{array}{c}
n-1 \\
k
\end{array}\right)+(1-o(1))\left(\begin{array}{l}
n-2 \\
k-2
\end{array}\right) .
$$

Recently, Glebov, Persons and Weps settled the 3-uniform case.

Theorem 2.2 ([3]) There exists an $n_{0}$ such that for any $n \geq n_{0}$ the maximum number of edges in a $k$-uniform hypergraph containing no hamiltonian $(k-1)$-cycle is $\left(\begin{array}{c}n-1 \\ 3\end{array}\right)+n-1$ if $3 \mid n-1$, and $\left(\begin{array}{c}n-1 \\ 3\end{array}\right)+n-2$ otherwise. 
The exact value is still unknown for $k \geq 4$.

\section{Shorter paths}

In [4] we try to generalize result of Gallai for hypergraphs.

Theorem 3.1 (Erdös-Gallai[1]) Let $G$ be a graph on $n$ vertices containing no path of length $k$. Then $e(G) \leq \frac{1}{2}(k-1) n$. Equality holds iff $G$ is the disjoint union of complete graphs on $k$ vertices.

We can get quite exact results regarding hypergraphs avoiding $(k-1)$-tight paths.

Theorem $3.2([4])$ Let $\mathcal{H}$ be an extremal $k$-uniform hypergraph containing no $(k-1)$-tight path of length $s$. Then

$$
(1+o(1)) \frac{s-1}{k}\left(\begin{array}{c}
n \\
k-1
\end{array}\right) \leq|e(\mathcal{H})| \leq(s-1)\left(\begin{array}{c}
n \\
k-1
\end{array}\right)
$$

However, if we use a different definition, then we can obtain sharper results.

Definition 3.3 Fix $k \geq 2$ and $t, 1 \leq t \leq k-1$. A Berge path of length $s$ in a hypergraph is a collection of $s$ hyperedges $h_{1}, \ldots, h_{s}$ and $s+1$ vertices $v_{1}, \ldots, v_{s+1}$ such that for each $1 \leq i \leq s$ we have $v_{i}, v_{i+1} \in h_{i}$.

$A$ t-tight Berge-path of length $s$ in a $k$-uniform hypergraph is a Berge-path on $s+1$ vertices $\left\{v_{1}, v_{2}, \ldots, v_{s+1}\right\}$ and s hyperedges $\left\{h_{1}, h_{2}, \ldots, h_{s}\right\}$ such that consecutive hyperedges intersect in at least $t$ points.

So one the difference between the two definitions is that the consecutive edges must intersect in exactly $t$ vertices or at least $t$ vertices. The other difference is that in this definition a path must contain a different edge for each $v_{i}, v_{i+1}$ along the path, which is not required in the previous definition. Note that if $t=1, k=2$ then both definitions gives the same as the usual definition for graphs.

Theorem 3.4 ([4]) Fix $k \geq 2$ and $t, 1 \leq t \leq k-1$. Fix s large. Let $\mathcal{H}$ be an extremal $k$-uniform hypergraph on $n$ vertices containing no t-tight Berge-path of length s. Then

$$
(1+o(1)) \frac{\left(\begin{array}{l}
n \\
t
\end{array}\right)\left(\begin{array}{l}
s \\
k
\end{array}\right)}{\left(\begin{array}{l}
s \\
t
\end{array}\right)} \leq e(\mathcal{H}) \leq \frac{\left(\begin{array}{l}
n \\
t
\end{array}\right)\left(\begin{array}{l}
s \\
k
\end{array}\right)}{\left(\begin{array}{l}
s \\
t
\end{array}\right)} .
$$

Using this result in [3] the authors give an asymptotically tight bound for the maximum number of edges in a hypergraph that doesn't contain a 
$(k-1)$-cycle.

In [4] more open problems are raised.

\section{Acknowledgment}

Parts of the work reported in the paper has been developed in the framework of the project "Talent care and cultivation in the scientific workshops of BME" project. This project is partially supported by the grant TÁMOP - 4.2.2.B10/1-2010-0009 and Hungarian National Research Fund OTKA (Grant Number 78439).

\section{References}

[1] P. Erdős, And T. Gallai, On maximal paths and circuits of graphs. Acta Math. Acad. Sci. Hungar. (1959) 10: 337-356

[2] P. Frankl and G.Y. Katona Extremal $k$-edge hamiltonian hypergraphs. Discrete Math. (2008) 308(8): 1415-1424

[3] R. Glebov, Y. Person and W. Weps, On extremal hypergraphs for Hamiltonian cycles. European J. Combin. (2012) 33(4): 544-555

[4] E. GyőRi, G.Y. Katona, N. Lemmons, Hypergraph Extensions of the ErdősGallai Theorem. Electronic Notes in Discrete Mathematics (2010) 36: 655-662

[5] G.Y. Katona and H.A. Kierstead, Hamiltonian chains in hypergraphs. J. Graph Theory (1999) 30(3): 205-212

[6] V. Rödl, A. Ruciński and E. Szemerédi, A Dirac-type theorem for 3uniform hypergraphs. Combin. Probab. Comput. (2006) 15(1-2):229-251

[7] V. RöDl, A. Ruciński And E. Szemerédi, An approximate Dirac-type theorem for $k$-uniform hypergraphs. Combinatorica (2008) 28(2):229-260

[8] V. RödL, A. Ruciński And E. Szemerédi, Dirac-type conditions for hamiltonian paths and cycles in 3-uniform hypergraphs. Adv. Math. (2011) 227(3): 1225-1299

[9] Zs. TuzA, Steiner systems and large non-hamiltonian hypergraphs. Le Matematiche (2006) 61(1):179-183 